\title{
Low-cooling-rate freezing in biomolecular cryo-electron microscopy for recovery of initial frames
}

\section{Research Article}

Cite this article: Wu C, Shi H, Zhu D, Fan K, Zhang $X$ (2021). Low-cooling-rate freezing in biomolecular cryo-electron microscopy for recovery of initial frames. QRB Discovery, 2: e11, 1-7 https://doi.org/10.1017/grd.2021.8

Received: 18 June 2021

Revised: 20 August 2021

Accepted: 01 September 2021

\section{Keywords:}

cryo-EM sample preparation; beam-induced motion; cooling rate; high temperature freezing

Author for correspondence:

*Xinzheng Zhang,

E-mail: xzzhang@ibp.ac.cn

Chunling Wu and Huigang Shi contributed equally to this work. (c) The Author(s) 2021. Published by Cambridge University Press. This is an Open Access article, distributed under the terms of the Creative Commons Attribution-NonCommercialNoDerivatives licence (https:// creativecommons.org/licenses/by-nc-nd/4.0/), which permits non-commercial re-use, distribution, and reproduction in any medium, provided the original work is unaltered and is properly cited. The written permission of Cambridge University Press must be obtained for commercial re-use or in order to create a derivative work.

\section{CAMBRIDGE} UNIVERSITY PRESS

\section{Chunling Wu ${ }^{1,2} \mathbb{D}$, Huigang Shi ${ }^{1,2} \mathbb{D}$, Dongjie $\mathrm{Zhu}^{3}$ (D), Kelong Fan $^{4} \mathbb{D}^{\mathbb{D}}$ and Xinzheng Zhang ${ }^{1,2 *}$ (D)}

\footnotetext{
${ }^{1}$ National Laboratory of Biomacromolecules, CAS Center for Excellence in Biomacromolecules, Institute of Biophysics, Chinese Academy of Sciences (CAS), Beijing 100101, P.R. China; ${ }^{2}$ College of Life Sciences, University of Chinese Academy of Sciences, Beijing 100049, P.R. China; ${ }^{3}$ School of Life Sciences, University of Science and Technology of China, Hefei 230026, P.R. China and ${ }^{4}$ CAS Engineering Laboratory for Nanozyme, Key Laboratory of Protein and Peptide

Pharmaceutical, Institute of Biophysics, Chinese Academy of Sciences (CAS), Beijing 100101, P.R. China
}

\begin{abstract}
When biological samples are first exposed to electrons in cryo-electron microcopy (cryo-EM), proteins exhibit a rapid 'burst' phase of beam-induced motion that cannot be corrected with software. This lowers the quality of the initial frames, which are the least damaged by the electrons. Hence, they are commonly excluded or down-weighted during data processing, reducing the undamaged signal and the resolution in the reconstruction. By decreasing the cooling rate during sample preparation, either with a cooling-rate gradient or by increasing the freezing temperature, we show that the quality of the initial frames for various protein and virus samples can be recovered. Incorporation of the initial frames in the reconstruction increases the resolution by an amount equivalent to using $\sim 60 \%$ more data. Moreover, these frames preserve the high-quality cryo-EM densities of radiation-sensitive residues, which is often damaged or very weak in canonical three-dimensional reconstruction. The improved freezing conditions can be easily achieved using existing devices and enhance the overall quality of cryo-EM structures.
\end{abstract}

\section{Introduction}

Biological molecules are structurally damaged when they are irradiated by electrons during electron microscopy. Embedding the samples in ice and imaging them at low temperatures suppresses the damage (Taylor and Glaeser, 1976; Hayward and Glaeser, 1979). Plunge-freezing thin layers of aqueous suspensions of proteins in liquid ethane embeds the proteins in vitreous ice (Adrian et al., 1984). This requires rapid cooling of the suspensions. Therefore, it has been recommended that the temperature should be maintained just above the melting point of liquid ethane at $-183^{\circ} \mathrm{C}$ (Passmore and Russo, 2016) to provide the highest cooling rate.

During cryo-electron microcopy (cryo-EM) imaging of proteins embedded in vitreous ice, beam-induced motion (BIM) occurs with high-energy electrons (Henderson et al., 2011; Brilot et al., 2012; Campbell et al., 2012). BIM is characterised by an initial rapid 'burst' phase (first 3-5 $\mathrm{e}^{-} / \AA^{2}$ ), followed by a slower phase (Glaeser, 2016; Ripstein and Rubinstein, 2016). BIM blurs the cryo-EM images, which reduces the resolution of the reconstruction. Using the movie mode of a direct detector device for imaging, a significant proportion of the BIM in the slower phase can be corrected by translational alignment of the movie frames (Campbell et al., 2012; Rubinstein and Brubaker, 2015; Zheng et al., 2017; Zivanov et al., 2019). However, the initial rapid burst motion cannot be effectively corrected with current software. Therefore, the initial frames remain blurred, which, unfortunately, contain the most structural information on the target proteins with the least amount of radiation damage. This is considered a key outstanding problem in cryo-EM (Vinothkumar and Henderson, 2016; Henderson and Russo, 2019), and frames that exhibit the effects of the rapid 'burst' phase are either excluded or down-weighted during data processing. In addition, it has been observed that electron bombardment of a cryoEM sample caused drum-like motion (Brilot et al., 2012). This caused both translational and rotational motion of a protein (Henderson et al., 2011; Brilot et al., 2012; Naydenova et al., 2020), among which the rotational motion cannot be corrected with existing motion correction software.

It has been suggested that the electron beam releases stress in the vitreous ice, which then causes the rapid burst motion. Different hypotheses exist for the origin of the stress. The beam may cause internal pressures to build up in the ice (Glaeser, 2008; Glaeser, 2016). The charge from the accumulation of secondary electrons on the sample surface could also blur the images (Henderson, 1992; Glaeser and Downing, 2004; Russo and Henderson, 2018). Additionally, 
beam-induced wrinkles in the grid carbon at low temperature may be a significant source of stress (Wright et al., 2006; Brilot et al., 2012), which has led to improved support films (Rhinow and Kühlbrandt, 2008; Yoshioka et al., 2010; Russo and Passmore, 2014a, 2014b; Huang et al., 2020). The stress may also be generated during vitrification and subsequent cooling (Glaeser, 2016). Thorne (2020) proposed that the cooling rate of the grid bars is significantly lower than that of the aqueous solution. Therefore, when the temperature of the solution approaches that of the liquid ethane, a further decrease in the temperature of grid bar causes shrinkage and stress in the ice. Recently, Naydenova et al. (2020) improved gold film fabrication by generating nanoscale arrays of $260-\mathrm{nm}$ holes. BIM is prevented because the ice in these small holes cannot be bent by stress. They proposed that rapid cooling causes compressive strain in the aqueous film. Wieferig et al. (2021) found that an extra step of ice devitrification also reduced the rapid burst motion. Because experimental data are lacking with regard to the stress origin, it is unclear how it could be reduced.

Here, reconstructions of various samples that were frozen at different cooling rates indicate that a lower rate helps to recover the quality of the initial frames. Simple modifications to existing protocols allow plunge-freezing at the lowest cooling rate required for vitrification, which enables the initial imaging frames to be recovered.

\section{Results}

\section{Vitreous ice on a grid frozen with a cooling-rate gradient}

To prevent crystalline ice from forming in the water, a high cooling rate is required to vitrify a thin aqueous film for cryo-EM sample preparation. Crystalline ice is a stable phase with the lowest energy. Therefore, when water is frozen at a low cooling rate, the molecules have more time to migrate to equilibrium positions, which may produce less stress. However, it is challenging to measure the absolute cooling rate of a thin aqueous film. Therefore, our goal was to produce a cooling-rate gradient on the grid.

Grids generally have a thick metal grating and a nanometerthick support film. During freezing, the grid was held by tweezers and plunged into liquid ethane. The tweezers transferred heat directly to the liquid ethane, or through the metal ring (shown in orange in Fig. 1a), which lowered the cooling rate near the tweezer tips and the metal ring (Vinothkumar and Henderson, 2016). The cooling rate of the aqueous film was thus higher in the region farthest from the tips and the metal ring, which led to a coolingrate gradient on the grid. To increase the gradient, we moved the tweezers approximately $0.5 \mathrm{~mm}$ towards the center of grid (Fig. 1a), which increased the contact between the tweezers and the grid and led to more heat transfer from the tweezers. As a result, we repeatedly found that crystalline ice formed in the mesh surrounding the position that was originally occupied by the tips of the tweezers (shown in dark blue in Fig. 1a), even in holes that had a very thin layer of ice (Fig. 1b). The ice in the mesh $\sim 0.7 \mathrm{~mm}$ away from this region and the metal ring (shown in light blue in Fig. 1a) was vitrified. Both crystalline and vitreous ice formed in the mesh between these two regions (shown in pink in Fig. 1a). We attributed crystalline ice formation to the low cooling rate. The cooling rate increased with distance from the tweezer tips or the metal ring. Therefore, in the pink region in Fig. $1 a$, the vitreous ice near the crystalline ice was frozen at the lower cooling rate.

\section{Initial frame recovery with low-cooling-rate freezing}

To verify whether the low cooling rate enabled recovery of the initial frames, cryo-EM datasets were acquired (Methods and Table S1) for different samples in the pink region. Samples such as apo-ferritin, glutamate dehydrogenase (GDH), and EV71 viruslike particles (VLP) were imaged with a Titan Krios electron microscope, equipped with a $\mathrm{K} 2$ camera for frame exposures of $1.2 \mathrm{e}^{-} / \AA^{2}$. Micrographs of samples in vitreous and crystalline ice could be easily distinguished via Fourier transforms, because the polycrystalline ice had strong diffraction rings at approximately 1/3.6 $\AA^{-1}$, as shown in Fig. $1 b$. Micrographs of proteins in a thin layer of vitreous ice were selected for further data processing. The resolutions of the per-frame reconstructions (see Methods) were calculated for each dataset. The overall difference in resolution among these datasets was because of different numbers of particles, and was not relevant to the recovery of rapid burst motion. The effect of recovering rapid burst motion was characterised by reducing the differences in resolution between the first few frames and that of the best frame in the same dataset. As shown in Table 1, and by the green lines in Fig. $2 a$, the resolutions of the per-frame reconstructions from the initial frames were effectively recovered (a)

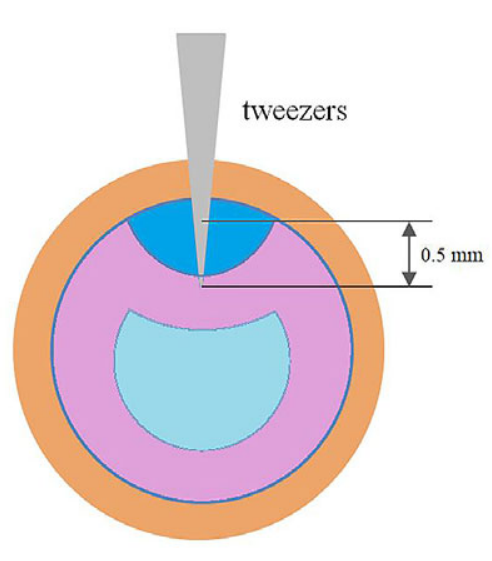

(b)
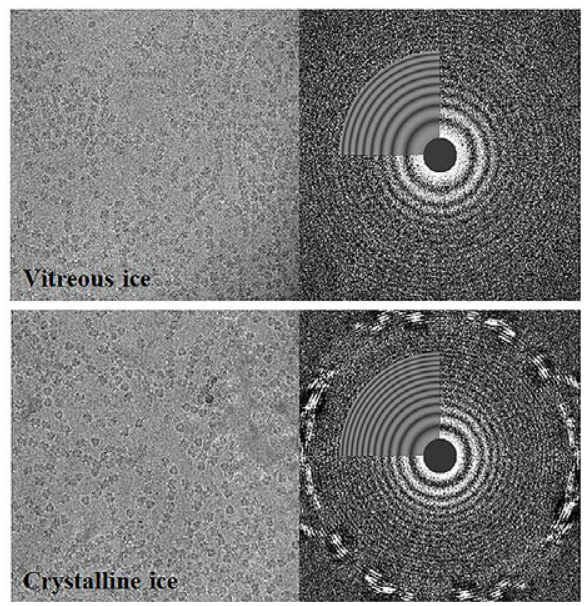

Fig 1. Cooling-rate gradient for cryo-electron microscopy (EM) samples. (a) Schematic of types of ice formed on grid covered by a cooling-rate gradient. The mesh in the dark blue region contained mostly crystalline ice. The pink region contained either vitreous ice or crystalline ice, and the light blue region contained mostly vitreous ice. $(b)$ glutamate dehydrogenase $(\mathrm{GDH})$ embedded in vitreous ice and crystalline ice frozen at $-183^{\circ} \mathrm{C}$. 
Table 1. Comparisons of various samples frozen under different conditions

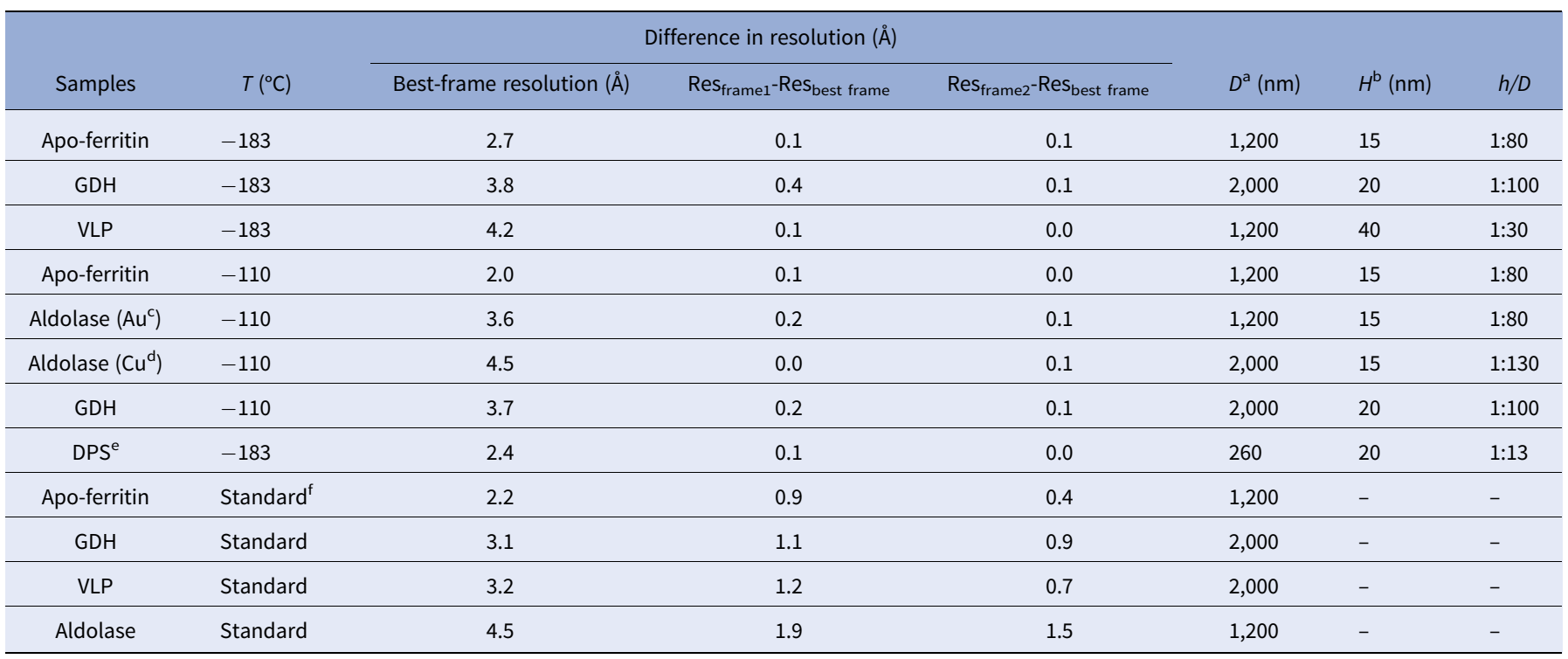

Abbreviations: DPS, DNA protection protein during starvation; GDH, glutamate dehydrogenase; VLP, virus-like particles.

${ }^{\mathrm{a}} D$ is the diameter of the grid holes.

$\mathrm{b} h$ is the thickness of the ice.

${ }^{\mathrm{C}} \mathrm{Au}$ means that the aldolase was collected in holey NiTi film covered on the Au grid.

${ }^{d} \mathrm{Cu}$ means that the aldolase was collected in holey carbon film covered on the Cu grid.

${ }^{\mathrm{e}}$ DPS dataset was reconstructed based on the deposited maps and mask by Naydenova et al. (2020). The DPS was frozen by regular plunge-freezing at $-183^{\circ} \mathrm{C}$ without intentionally moving the tweezers using a grid with 260-nm-diameter holes.

${ }^{f}$ Standard means using regular freezing conditions at $-183^{\circ} \mathrm{C}$, without intentionally moving the tweezers.

(a)

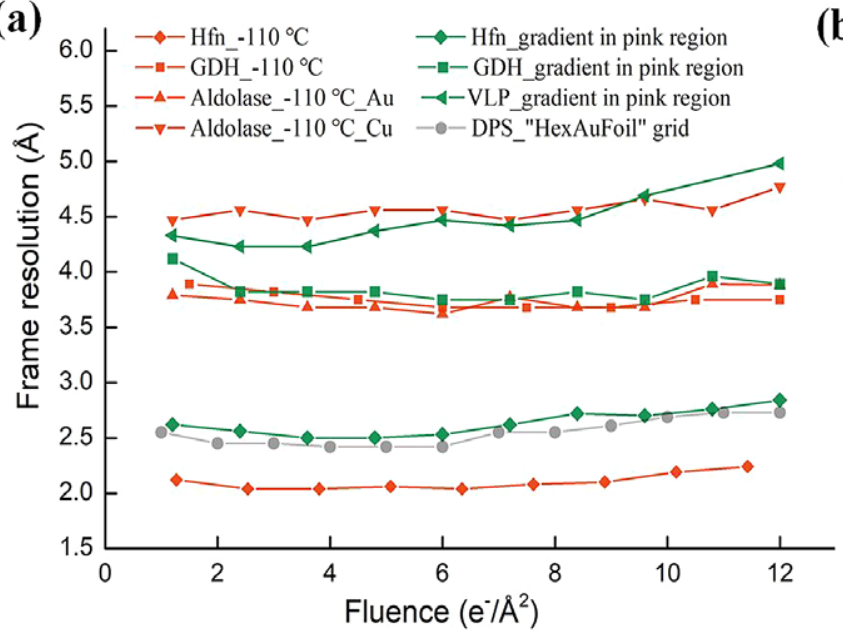

(b)

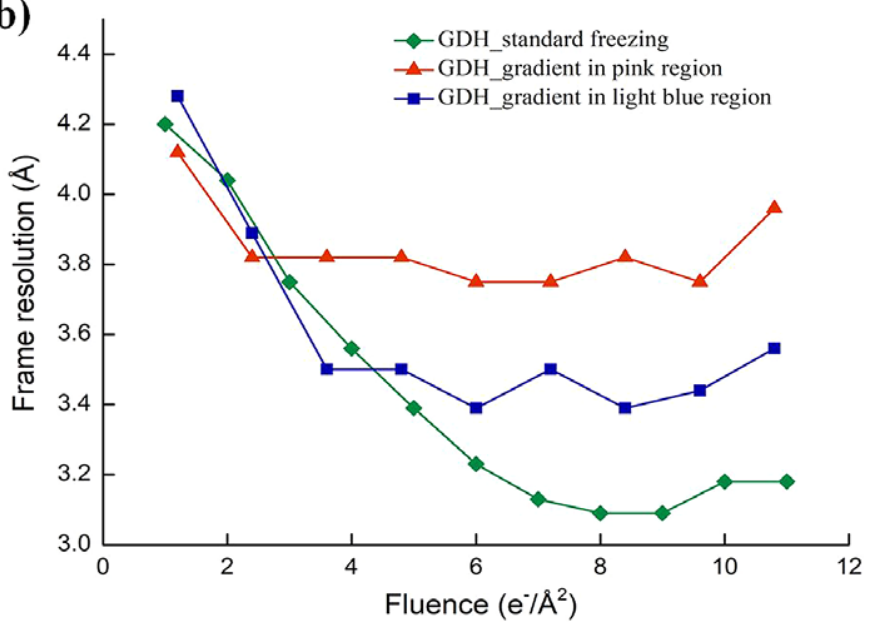

Fig. 2. Resolutions of per-frame reconstructions for various samples frozen at different cooling rates. (a) Resolutions of per-frame reconstructions of various samples frozen by lowering the cooling rate. The green lines are for apo-ferritin (rhombus), glutamate dehydrogenase (GDH; square) and virus-like particles (VLP; triangle) collected in the pink region marked in Fig. 1a. The red lines are for apo-ferritin (rhombus), GDH (square) and aldolase (triangle) frozen at $-110^{\circ} \mathrm{C}$. Aldolase was frozen on an Au grid covered with a holey NiTi film (regular triangles), and also on a Cu grid covered with a holey carbon film (inverted triangle). The grey line shows the resolutions of downloaded per-frame reconstructions (EMD11210) of DNA protection protein during starvation (DPS). (b) Resolutions of per-frame reconstructions of GDH datasets using a cooling-rate gradient. The green dataset was collected for a standard frozen sample formed at the highest cooling rate, the blue dataset was collected in the light-blue region in Fig. 1a, and the red dataset was collected in the pink region in Fig. 1a.

for all three samples. The first two per-frame reconstructions of VLP with $0-1.2$ and $1.2-2.4 \mathrm{e}^{-} / \AA^{2}$ exposures exhibited the best resolutions. The differences in resolution between the first and best per-frame reconstruction of apo-ferritin and GDH were 0.1 and $0.4 \AA$, and that between the second and best per-frame reconstruction was 0.1 and $0.1 \AA$, respectively (Table 1 ). For comparison, the resolutions of the per-frame reconstructions from the first two frames of the same samples after normal rapid freezing were $\sim 1 \AA$ lower than that for the best frame, as shown in Table 1 and Fig. S1a-c.
Thus, the initial frames were almost fully recovered in the pink region frozen at the lower cooling rate.

To test whether freezing at a higher cooling rate led to decreased resolution in the initial frames, we collected GDH data in the light blue region (Fig. 1a) on the same grid, with a frame exposure of $1.2 \mathrm{e}^{-} / \AA^{2}$. Micrographs of proteins in a thin layer of vitreous ice were selected for further data processing. As shown by the blue line in Fig. $2 b$, the resolutions of the initial frames collected in the light blue region were not restored as well as the data collected in the pink 

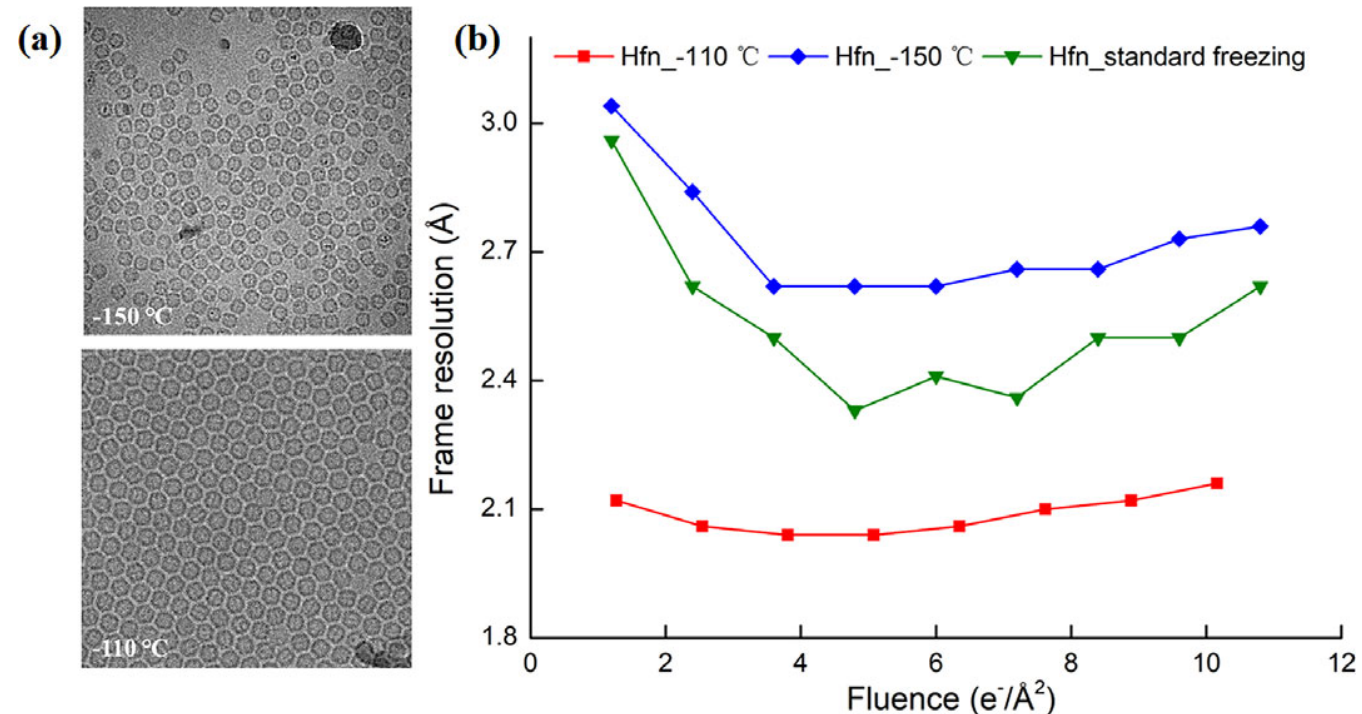

Fig. 3. Effect of freezing temperature. (a) Images of apo-ferritin embedded in vitreous ice frozen at -150 and $-110^{\circ} \mathrm{C}$. (b) Resolutions of per-frame reconstructions of apo-ferritin frozen at $-183^{\circ} \mathrm{C}$ (green), $-150^{\circ} \mathrm{C}$ (blue) and $-110^{\circ} \mathrm{C}$ (red), with exposures of $1.2,1.2$ and $1.27 \mathrm{e}^{-} / \AA^{2}$ for each frame, respectively.

region. However, when the data collected in the light blue region were compared with data from the sample frozen under normal freezing conditions without moving the tweezers, the differences in resolution between the initial and best per-frame reconstructions in the former were small. This indicated that the initial frames collected in the light blue region were only partially recovered.

\section{Initial frame recovery with increased freezing temperature}

In routine cryo-EM sample preparation, the freezing temperature is $-183{ }^{\circ} \mathrm{C}$ to maximise the formation of vitreous ice. Higher temperatures would reduce the cooling rate (Ryan et al., 1987), and may still produce vitreous ice. To determine the highest vitrification temperature for cryo-EM samples, apo-ferritin was frozen by plunging grids into liquid ethane at temperatures ranging from $-90^{\circ} \mathrm{C}$ to $-183^{\circ} \mathrm{C}$, using automated devices (Gatan CP3). Because the tweezers were not adjusted, less heat was conducted from the tweezers to the metal ring, resulting in more homogeneous cooling rates across the grid. We used a thermocouple calibrated by measuring liquid nitrogen and the melting points of five substances at atmospheric pressure (Fig. S2) to calibrate the liquid ethane temperature in both the CP3 and EMGP plunging devices (Table S2). The experiments revealed that thin aqueous samples of apo-ferritin, $\mathrm{GDH}$, aldolase and $\beta$-galactosidase were vitrified in liquid ethane at calibrated temperatures below $-110{ }^{\circ} \mathrm{C}$ (Fig. S3). Most of the samples were crystalline above $-110^{\circ} \mathrm{C}$.

For apo-ferritin samples frozen at $-183,-150$ and $-110^{\circ} \mathrm{C}$, we acquired datasets with a Titan Krios microscope equipped with a K2 camera (Fig. 3a). The apo-ferritin in the three datasets was reconstructed to $2.1,2.4$ and $1.9 \AA$, respectively, using standard data processing via single-particle analysis (see Methods). The resolutions of the per-frame reconstructions from the three datasets are shown in Fig. $3 b$. At $-183^{\circ} \mathrm{C}$, the resolutions of the first three per-frame reconstructions were significantly lower than those of subsequent per-frame reconstructions. This difference became smaller with increased freezing temperature. At $-110{ }^{\circ} \mathrm{C}$, the per-frame reconstruction for the second frame had the best resolution, and the resolution of the first per-frame reconstruction was slightly lower than that of the second.
To support our observations, we collected cryo-EM datasets for GDH and aldolase on two different grids (Table 1 and Table S1) vitrified at $-110^{\circ} \mathrm{C}$, with frame exposures of $1.5,1.2$ and $1.2 \mathrm{e}^{-} / \AA^{2}$, respectively. The per-frame reconstructions are shown in Fig. $2 a$ (red lines). As shown in Table 1, the first two frames were effectively recovered in all the datasets, which indicated that freezing at a lower cooling rate by increasing the freezing temperature restored the resolution of the initial frames. Conversely, the corresponding resolutions of per-frame reconstructions from the initial frames of samples frozen under standard freezing conditions were significantly lower than those of subsequent frames, as shown in Table 1 and Fig. S1d-f.

\section{Benefits of recovering the initial frames}

Certain protein amino-acid residues that contain carboxyl sidechain groups, solvent-exposed disulfide bonds or are part of an enzyme active site, are highly sensitive to electron damage (Weik et al., 2000; Bartesaghi et al., 2015; Glaeser, 2016). As shown in Fig. 4, we compared the EM map densities of site-specific amino acids in different per-frame reconstructions. Side-chain densities of damage-sensitive amino acids were well-preserved in the first two per-frame reconstructions $\left(0-2.54 \mathrm{e}^{-} / \AA^{2}\right)$, but were already damaged in the fourth frame. The cumulative electron dose before acquiring the fourth frame was $3.8 \mathrm{e}^{-} / \AA^{2}$.

After full frames were processed with a dose-weighting procedure in MotionCor2 (full-frames dataset), the apo-ferritin sample frozen at $-110^{\circ} \mathrm{C}$ had an overall resolution of $1.9 \AA$. To compare the quality of these reconstructions with a standard cryo-EM sample affected by rapid-burst BIM, we mimicked the data by excluding the first three frames from the dataset frozen at $-110^{\circ} \mathrm{C}$ during the dose-weighting procedure. For the same apo-ferritin particles, the resulting partial-frames dataset had an overall resolution of 2.0 A. The ResLog (Stagg et al., 2014) plots from these two datasets indicated that the partial-frames dataset mimicking cryo-EM samples at $-183{ }^{\circ} \mathrm{C}$ required $60 \%$ more particles to achieve the same resolution as that for the full-frames dataset acquired at $-110{ }^{\circ} \mathrm{C}$ (Fig. 5). Moreover, we observed a slight improvement in the b-factor in the full-frames dataset. The full-frames dataset exhibited 


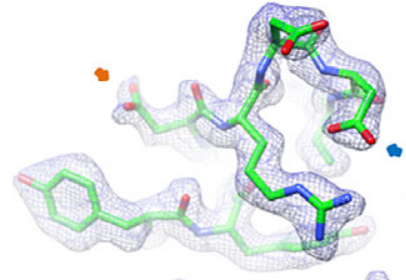

Frame1 0 1.27e-/ $\AA^{2}$

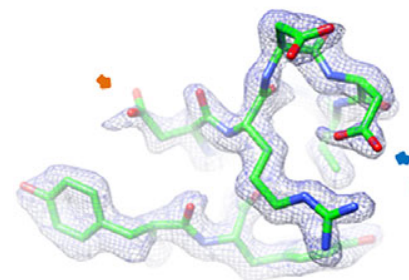

Frame4 3.81 5.08 e-/ $\AA^{2}$

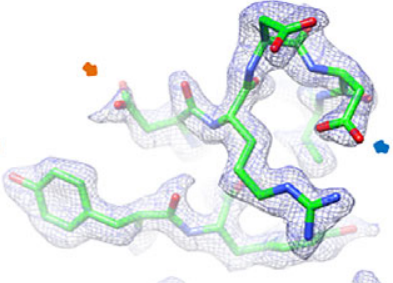

Frame2 1.27 2.54 e-/ ${ }^{2}$

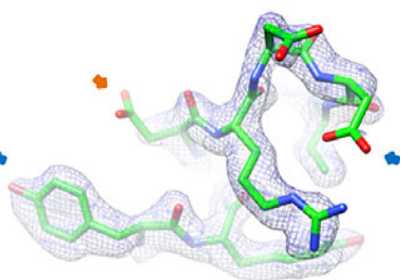

Frame8 8.89 10.16 e-/ $\AA^{2}$
Fig. 4. Maps showing the side chain densities of the initial per-frame reconstructions of apo-ferritin frozen at $-110^{\circ} \mathrm{C}$.

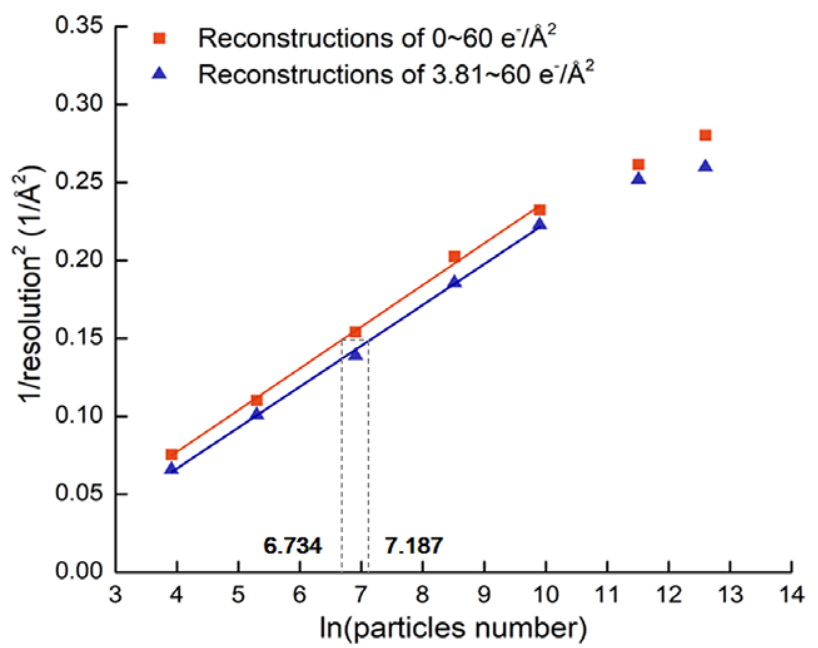

Fig. 5. ResLog plots of datasets including or excluding the first three frames. The red and blue straight lines were fit from the first five data points, and correspond to the datasets with or without the first three frames, respectively. The b-factors of the fullframes dataset and partial-frames dataset were $74.9 \AA^{2}$ and $76.3 \AA^{2}$, respectively. The length between the two horizontal dashed lines is 0.453 , which indicates that the reconstruction of the dataset without the first three frames needed $\sim 1.6$ times particles to achieve the same resolution as that for the dataset with the first three frames.

higher image quality than that of the partial-frames dataset because the former was averaged with three extra frames having the least amount of electron radiation damage. The higher image quality enabled a more accurate alignment in the refinement during data processing, thus improving the b-factor.

\section{Discussion}

The $-110{ }^{\circ} \mathrm{C}$ vitrification temperature differs from previous observations that cubic ice formed on microdroplets at $-135{ }^{\circ} \mathrm{C}$ (Dubochet and McDowall, 1981; Dubochet et al., 1982). However, the 30-nm-thick aqueous layer in our experiments was much smaller than a microdroplet, which led to a much higher cooling rate that produced vitrification. It has been calculated and observed that the transition temperature from vitreous to crystalline ice for pure water is approximately $-135^{\circ} \mathrm{C}$ (Dowell and Rinfret, 1960; Dubochet and McDowall, 1981). However, solution components such as salts and proteins increase the glass transition temperature (Mackenzie, 1977; Gilkey and Staehelin, 1986; Warkentin et al., 2013; Thorne, 2020). Rash (1983) have suggested that vitrified biological samples must be kept below $-100{ }^{\circ} \mathrm{C}$ to prevent changes in the physical state. Wieferig et al. (2021) also used temperatures between -110 and $-100{ }^{\circ} \mathrm{C}$ to observe a phase transition of an aqueous film from vitreous to crystalline ice.

Some of the reports on reducing BIM via freezing proteins at $-110{ }^{\circ} \mathrm{C}$ have been online at BioRxiv since October 2019 (https:// doi.org/10.1101/824698). Our data showed that the rapid burst motion was related with the freezing temperature, which indicates that the stress in vitreous ice caused by the rapid freezing may play important role in the rapid burst motion. Naydenova et al. (2020) proposed that rapid cooling causes compressive strain in the thin film, which agreed with our work. They showed that, using HexAuFoil grids, the quality of the first several per-frame reconstructions of a $223-\mathrm{kDa}$ DNA protection protein during starvation (DPS) were recovered. We calculated the resolutions of the perframe reconstructions based on their deposited maps and mask (EMD-11210), as shown by the grey line in Fig. $2 a$ and in Table 1. The quality of the first frame for DPS was substantially recovered, but was still slightly worse than that of the second frame based on a 0.143 FSC criterion, as observed for other samples using our freezing methods (Fig. 2a). As shown in Table 1, the qualities of the initial frames were almost fully recovered, which indicated that both lowcooling-rate methods worked similarly to the HexAuFoil grid method.

Naydenova et al. (2020) used standard freezing conditions to vitrify the sample. To prevent bending of the ice by the stress release during imaging, they made the thickness of the ice $(h)$, divided by the diameter $(D)$ of the grid holes, $\geq 1 / 11$. Thus, the DPS was frozen using a grid with 260-nm diameter holes. Here, where the cooling rate was lowered to reduce the stress in the ice, $h / D$ was not limited, as shown in Table 1. Therefore, various grids with different hole sizes could be used. Using large holes during imaging prevents recording of the supporting film, which significantly improves the throughput of the data collection.

Because the cooling rates of the tweezers, grid bars and metal ring are still lower than that of aqueous film in a standard cryoEM sample preparation, a cooling-rate gradient always occurs on the grid (Vinothkumar and Henderson, 2016; Thorne, 2020). The magnitude of the gradient depends on how much heat is transferred from the tweezers to the grid. Freezing at $-183^{\circ} \mathrm{C}$ with the position of the tweezers adjusted to make more contact with the grid results in a large cooling-rate gradient. Although the region with a sufficiently low cooling rate (pink region in Fig. 1a) was large enough for data collection, freezing at $-110^{\circ} \mathrm{C}$ enabled data collection over the whole grid. Therefore, freezing at the higher temperature is preferred. Because of errors in measuring the liquid ethane temperature when using different plunging devices, a combination of freezing at slightly lower than $-110^{\circ} \mathrm{C}$, with less tweezers adjustment, may create a smaller cooling-rate gradient that enlarges the area containing rapid motion-free proteins.

\section{Materials and methods}

\section{Protein and cryo-EM sample preparation}

Human apo-ferritin was diluted to a concentration of $\sim 2 \mathrm{mg} / \mathrm{ml}$ and purified according to previous reports (Fan et al., 2012; 
Jiang et al., 2020). The apo-ferritin proteins were expressed in Escherichia coli, where they self-assembled into a 24-subunit nanocage. They were purified via size-exclusion chromatography on a Sepharose 6 PG XK 16/70 column from GE Healthcare (Livonia, MI, USA), followed by ion-exchange chromatography on a Q-Sepharose Fast Flow from GE Healthcare (Livonia, MI, USA). The apo-ferritin concentration was determined in triplicate with a BCA protein assay kit (Pierce) form Thermo Fisher Scientific (Portage, MI, USA), using bovine serum albumin as the standard. The GDH from Sigma-Aldrich (Milwaukee, WI, USA) was dialysed in 100-mM potassium phosphate ( $\mathrm{pH}$ 6.8) overnight prior to purification via gel filtration. The GDH concentration in the cryo-EM samples was $\sim 3 \mathrm{mg} / \mathrm{ml}$. Aldolase was purchased from Sigma-Aldrich (Milwaukee, WI, USA) and solubilised in 20-mM HEPES at $\mathrm{pH} 7.5$ and $50-\mathrm{mM} \mathrm{NaCl}$. It was then purified via gel filtration using a Superdex 200 increase 10/300 from GE Healthcare (Livonia, MI, USA) column equilibrated in $20 \mathrm{mM}$ HEPES at $\mathrm{pH} 7.5$ and $50-\mathrm{mM} \mathrm{NaCl}$. Peak fractions were pooled and concentrated to $2 \mathrm{mg} / \mathrm{ml}$. The VLP was a gift from Dr. Yingzhi Xu (Minhai Biotechnology Co.) and was diluted to $\sim 4 \mathrm{mg} / \mathrm{ml}$ with $20 \mathrm{mM}$ Tris. $\mathrm{HCl}$ pH 7.5 and $150-\mathrm{mM} \mathrm{NaCl}$.

Approximately 3- $\mu$ l sample aliquots were applied to a glowdischarged holey NiTi film covering an Au grid, or to a carbon film covering a $\mathrm{Cu}$ grid. The grids were blotted for 3-7 s with an automated plunging device CP3 from Gatan (Warrendale, PA, USA) or EMGP from Leica (Wetzlar, Germany), and then flashplunged into liquid ethane at various temperatures ranging from -90 to $-183^{\circ} \mathrm{C}$ (details in Table S1).

\section{Data acquisition and processing}

Datasets of apo-ferritin frozen at $-183{ }^{\circ} \mathrm{C}$ and aldolase frozen at $-110{ }^{\circ} \mathrm{C}$ on Au grids (Table 1 ) were collected with a FEI Titan Krios form Thermo Fisher Scientific (Portage,MI, USA), equipped with a direct detector K2 summit from Gatan (Warrendale, PA, USA) with super-resolution mode, at a magnification of $165,000 \times$ and binned pixel sizes of $0.82 \AA$. The GDH datasets obtained in the pink and light-blue regions (Fig. 1a) of the Cu grid frozen at $-183^{\circ}$ $\mathrm{C}$ (Table 1) were imaged similarly, with a GIF quantum energy filter (width of $20 \mathrm{eV}$ ) by the beam-image shift data collection method (Wu et al., 2019). All the remaining datasets were collected similarly, with details listed in Table S1.

Images of a thin-layer aqueous sample were selected manually for further data processing. In all datasets, the BIM was corrected with MotionCor2 software (Zheng et al., 2017). Aligned stacks were saved for per-frame reconstructions. The defocus parameters were estimated with CTFFIND4 software (Rohou and Grigorieff, 2015). About 2,000 particles were picked by e2boxer.py semiautomatic particle-picking software (Tang et al., 2007), or by manual picking with RELION software, and were processed by two-dimensional (2D) classification. This yielded 2D averages that were used by RELION as references for automatic particle-picking in all micrographs (Scheres, 2012). After the reference-free 2D and 3D classifications, particles were selected and processed via automatic refinement. An extra round of refinement was performed after a CTFFIND4 refinement in RELION-3 (Zivanov et al., 2018). The reconstruction resolution was determined via post-processing in RELION, which used a soft mask for each dataset.

For per-fame reconstruction, a single frame was split from the aligned stacks written out with MotionCor2. The particles were reextracted in each frame image using parameters from the previous refinement iteration, and per-frame reconstructions were calculated from re-extracted particles via relion_reconstruct_mpi. The resolutions of the per-frame reconstructions were determined by post-processing in RELION, with the same soft mask used in the initial reconstruction of each sample.

\section{Atomic model refinement}

Protein data bank coordinates 1 MFR served as a starting model for building the atomic structure of apo-ferritin. Amino acids were mutated in COOT software (Emsley and Cowtan, 2004), according to the apo-ferritin amino acid sequence. Manual adjustments were made in COOT. The model was refined with Phenix software (Adams et al., 2010).

Acknowledgements. We thank Dr. Yingzhi Xu at Minhai Biotechnology Co. for supplying the VLP sample. We thank L. Kong for cryo-EM data storage and backup. Cryo-EM data collection was performed at the Center for Biological Imaging, Core Facilities for Protein Science at the Institute of Biophysics (IBP), Chinese Academy of Sciences (CAS). We thank X. J Huang, B. L. Zhu, X. J. Li, L. H. Chen, F. Sun and other staff members at the Center for Biological Imaging (IBP, CAS). The project was funded by the National Key R\&D Program of China (2017YFA0504700), the National Natural Science Foundation of China (31930069) the Strategic Priority Research Programme of the Chinese Academy of Sciences (XDB37040101) and the Key Research Programme of Frontier Sciences at the Chinese Academy of Sciences (ZDBS-LY- SM003). We thank Alan Burns for editing the language of a draft of this manuscript.

Supplementary Materials. To view supplementary material for this article, please visit http://dx.doi.org/10.1017/qrd.2021.8.

Author contributions X.Z. C.W. and H.S. designed the experiment. C.W. and H.S. performed the experiments. K.F prepared apo-ferritin sample. C.W. and H.S. prepared the cryo-EM specimen and performed data collection. C.W., H.S. and D.Z. performed data processing. C.W., H.S. and X.Z. analysed the result and wrote the manuscript. X.Z. supervised the whole experiment. All authors discussed and commented on the results and the manuscript.

Conflict of interest. The authors declare no conflict of interest.

Data availability statement. The deposit code of the frame reconstructions of apo-ferritin frozen at $-110^{\circ} \mathrm{C}$ is EMD-31736. The deposit code of the frame reconstructions of apo-ferritin frozen at $-183{ }^{\circ} \mathrm{C}$ with cooling rate gradient is EMD-31737. All other relevant data are available from the corresponding author upon reasonable request.

Open Peer Review. To view the open peer review materials for this article, please visit http://dx.doi.org/10.1017/qrd.2021.8.

\section{References}

Adams PD, Afonine PV, Bunkóczi G, Chen VB, Davis IW, Echols N, Headd JJ, Hung LW, Kapral GJ, Grosse-Kunstleve RW, McCoy AJ, Moriarty NW, Oeffner R, Read RJ, Richardson DC, Richardson JS, Terwilliger TC and Zwart PH (2010) PHENIX: a comprehensive Python-based system for macromolecular structure solution. Acta Crystallographica Section D: Biological Crystallography 66(2), 213-221.

Adrian M, Dubochet J, Lepault J and McDowall AW (1984) Cryo-electron microscopy of viruses. Nature 308(5954), 32.

Bartesaghi A, Merk A, Banerjee S, Matthies D, Wu X, Milne JL and Subramaniam S (2015) $2.2 \AA$ resolution cryo-EM structure of $\beta$-galactosidase in complex with a cell-permeant inhibitor. Science 348(6239), 1147-1151.

Brilot AF, Chen JZ, Cheng A, Pan J, Harrison SC, Potter CS, Carragher B, Henderson R and Grigorieff $\mathbf{N}$ (2012) Beam-induced motion of vitrified specimen on holey carbon film. Journal of Structural Biology 177(3), 630-637.

Campbell MG, Cheng A, Brilot AF, Moeller A, Lyumkis D, Veesler D, Pan J, Harrison SC, Potter CS, Carragher B and Grigorieff N (2012) Movies of ice- 
embedded particles enhance resolution in electron cryo-microscopy. Structure 20(11), 1823-1828.

Dowell LG and Rinfret AP (1960). Low-temperature forms of ice as studied by $X$-ray diffraction. Nature 188(4757), 1144-1148.

Dubochet J, Lepault J, Freeman R, Berriman J and Homo JC (1982) Electron microscopy of frozen water and aqueous solutions. Journal of Microscopy 128(3), 219-237.

Dubochet J and McDowall AW (1981) Vitrification of pure water for electron microscopy. Journal of Microscopy 124(3), 3-4.

Emsley P and Cowtan K (2004) Coot: model-building tools for molecular graphics. Acta Crystallographica Section D: Biological Crystallography 60(12), 2126-2132.

Fan K, Cao C, Pan Y, Lu D, Yang D, Feng J, Song L, Liang M and Yan X (2012) Magnetoferritin nanoparticles for targeting and visualizing tumour tissues. Nature Nanotechnology 7(7), 459.

Gilkey JC and Staehelin LA (1986) Advances in ultrarapid freezing for the preservation of cellular ultrastructure. Journal of Electron Microscopy Technique 3(2), 177-210.

Glaeser R (2016) Specimen behavior in the electron beam. In Methods in Enzymology, vol. 579. Amsterdam: Elsevier, pp. 19-50.

Glaeser RM (2008) Retrospective: radiation damage and its associated "information limitations". Journal of Structural Biology 163(3), 271-276.

Glaeser RM and Downing KH (2004) Specimen charging on thin films with one conducting layer: discussion of physical principles. Microscopy and Microanalysis 10(6), 790-796.

Hayward SB and Glaeser RM (1979) Radiation damage of purple membrane at low temperature. Ultramicroscopy $\mathbf{4}(2), 201-210$.

Henderson R (1992) Image contrast in high-resolution electron microscopy of biological macromolecules: TMV in ice. Ultramicroscopy 46(1-4), 1-18.

Henderson R, Chen S, Chen JZ, Grigorieff N, Passmore LA, Ciccarelli L, Rubinstein JL, Crowther RA, Stewart PL and Rosenthal PB (2011) Tilt-pair analysis of images from a range of different specimens in singleparticle electron cryomicroscopy. Journal of Molecular Biology 413(5), 1028-1046.

Henderson R and Russo CJ (2019) Single particle CryoEM: potential for further improvement. Microscopy and Microanalysis 25(S2), 4-5.

Huang X, Zhang L, Wen Z, Chen H, Li S, Ji G, Yin C and Sun F (2020) Amorphous nickel titanium alloy film: a new choice for cryo electron microscopy sample preparation. Progress in Biophysics and Molecular Biology 156, $3-13$.

Jiang B, Chen X, Sun G, Chen X, Yin Y, Jin Y, Mi Q, Ma L, Yang Y, Yan X and Fan K (2020) A natural drug entry channel in the ferritin nanocage. Nano Today 35, 100948.

Mackenzie AP (1977) Non-equilibrium freezing behaviour of aqueous systems. Philosophical Transactions of the Royal Society of London. B, Biological Sciences 278(959), 167-189.

Naydenova K, Jia P and Russo CJ (2020) Cryo-EM with sub-1 $\AA$ specimen movement. Science 370(6513), 223-226.

Passmore LA and Russo CJ (2016) Specimen preparation for high-resolution cryo-EM In Methods in Enzymology, vol. 579. Amsterdam: Elsevier, pp. 51-86.

Rash JE (1983) The rapid-freeze technique in neurobiology. Trends in Neurosciences 6, 208-212.

Rhinow D and Kühlbrandt W (2008) Electron cryo-microscopy of biological specimens on conductive titanium-silicon metal glass films. Ultramicroscopy 108(7), 698-705.

Ripstein Z and Rubinstein J (2016) Processing of cryo-EM movie data. In Methods in Enzymology, vol. 579. Amsterdam: Elsevier, pp. 103-124.
Rohou A and Grigorieff N (2015) CTFFIND4: fast and accurate defocus estimation from electron micrographs. Journal of Structural Biology 192(2), 216-221.

Rubinstein JL and Brubaker MA (2015) Alignment of cryo-EM movies of individual particles by optimization of image translations. Journal of Structural Biology 192(2), 188-195.

Russo CJ and Henderson R (2018) Charge accumulation in electron cryomicroscopy. Ultramicroscopy $\mathbf{1 8 7}, 43-49$.

Russo CJ and Passmore LA (2014a) Controlling protein adsorption on graphene for cryo-EM using low-energy hydrogen plasmas. Nature Methods 11(6), 649-652.

Russo CJ and Passmore LA (2014b) Ultrastable gold substrates for electron cryomicroscopy. Science 346(6215), 1377-1380.

Ryan K, Purse D, Robinson S and Wood J (1987) The relative efficiency of cryogens used for plunge—cooling biological specimens. Journal of Microscopy, 145(1), 89-96

Scheres SH (2012) A Bayesian view on cryo-EM structure determination. Journal of Molecular Biology 415(2), 406-418.

Stagg SM, Noble AJ, Spilman M and Chapman MS (2014) ResLog plots as an empirical metric of the quality of cryo-EM reconstructions. Journal of Structural Biology 185(3), 418-426.

Tang G, Peng L, Baldwin PR, Mann DS, Jiang W, Rees I and Ludtke SJ. (2007) EMAN2: an extensible image processing suite for electron microscopy. Journal of Structural Biology 157(1), 38-46.

Taylor KA and Glaeser RM (1976) Electron microscopy of frozen hydrated biological specimens. Journal of Ultrastructure Research 55(3), 448-456.

Thorne RE (2020) Hypothesis for a mechanism of beam-induced motion in cryoelectron microscopy. IUCrJ 7(3), 416-421.

Vinothkumar KR and Henderson R (2016) Single particle electron cryomicroscopy: trends, issues and future perspective. Quarterly Reviews of Biophysics 49, e13. https://doi.org/10.1017/S0033583516000068

Warkentin M, Sethna JP and Thorne RE (2013) Critical droplet theory explains the glass formability of aqueous solutions. Physical Review Letters 110(1), 015703.

Weik M, Ravelli RB, Kryger G, McSweeney S, Raves ML, Harel M, Gros P, Silman I, Kroon J and Sussman JL (2000) Specific chemical and structural damage to proteins produced by synchrotron radiation. Proceedings of the National Academy of Sciences 97(2), 623-628.

Wieferig J-P., Mills DJ and Kühlbrandt W (2021) Devitrification reduces beam-induced movement in cryo-EM. IUCrJ 8(2), 186-194.

Wright ER, Iancu CV, Tivol WF and Jensen GJ (2006) Observations on the behavior of vitreous ice at $\sim 82$ and $\sim 12 \mathrm{~K}$. Journal of Structural Biology 153 (3), 241-252.

Wu C, Huang X, Cheng J, Zhu D and Zhang X (2019). High-quality, highthroughput cryo-electron microscopy data collection via beam tilt and astigmatism-free beam-image shift. Journal of Structural Biology 208(3), 107396.

Yoshioka C, Carragher B and Potter C (2010) Cryomesh ${ }^{\mathrm{m}}$ : a new substrate for cryo-electron microscopy. Microscopy and microanalysis: the official journal of Microscopy Society of America, Microbeam Analysis Society, Microscopical Society of Canada 16(1), 43.

Zheng SQ, Palovcak E, Armache J-P., Verba KA, Cheng Y and Agard DA J. N. m. (2017) MotionCor2: anisotropic correction of beam-induced motion for improved cryo-electron microscopy. Nature Methods 14(4), 331.

Zivanov J, Nakane T, Forsberg BO, Kimanius D, Hagen WJ, Lindahl E and Scheres SH (2018) New tools for automated high-resolution cryo-EM structure determination in RELION-3. Elife 7, e42166.

Zivanov J, Nakane T and Scheres SH (2019) A Bayesian approach to beaminduced motion correction in cryo-EM single-particle analysis. IUCrJ 6(1), 5-17. 